\title{
The Wilson-Cowan model, 36 years later
}

\author{
Alain Destexhe · Terrence J. Sejnowski
}

Received: 26 April 2009 / Accepted: 6 July 2009 / Published online: 7 August 2009

(c) The Author(s) 2009. This article is published with open access at Springerlink.com

\begin{abstract}
The Wilson-Cowan model of interacting neurons (1973) is one of the most influential papers published in Biological Cybernetics (Kybernetik). This paper and a companion paper published in 1972 have been cited over 1000 times. Rather than focus on the microscopic properties of neurons, Wilson and Cowan analyzed the collective properties of large numbers of neurons using methods from statistical mechanics, based on the mean-field approach. New experimental techniques to measure neuronal activity at the level of large populations are now available to test these models, including optical recording of brain activity with intrinsic signals and voltage sensitive dyes, and new methods for analyzing EEG and MEG. These measurement techniques have revealed patterns of coherent activity that span centimetres of tissue in the cerebral cortex. Here the underlying ideas are reviewed in a historic context.

The Wilson-Cowan $(1972 ; 1973)$ model is a set of differential equations that describe the time evolution of the mean level of activity of populations of neurons, using the now familiar nonlinear sigmoidal function to represent the interactions between the populations. This mean-field approach has been used to address many problems in computational
\end{abstract}

This note was written by Alain Destexhe and Terry Sejnowski, the Editors-in-Chief of the Journal of Computational Neuroscience and Neural Computation, on the occasion of the appearance of Volume 100 of Biological Cybernetics.

\footnotetext{
A. Destexhe $(\varangle)$

91198 Gif-sur-Yvette, France

e-mail: alain.destexhe@unic.cnrs-gif.fr

T. J. Sejnowski

The Salk Institute/CNL, P.O. Box 85800,

San Diego, CA 92186-5800, USA
}

Unité de Neurosciences Intégratives et Computationnelles (UNIC),

Centre National de la Recherche Scientifique (CNRS), neuroscience (Sejnowski 1976a; Amit and Brunel 1997; Brunel 2000; Latham et al. 2000; Hertz et al. 2004; Renart et al. 2007). The Wilson-Cowan model has also been the starting point for many extensions. The special case with symmetric connectivity allowed an energy function to be defined and was analyzed by Hopfield (1982) and Hopfield and Tank (1986). They showed that the point attractors in this model could be used to store content-addressable memories and that constraint satisfaction problems could also be solved with these equations. Continuum extensions have also been proposed (van Hemmen 2004), where the differences with excitable media are analyzed.

Another important special case is the "balanced" network, in which the excitatory and inhibitory inputs on average cancel (Shadlen and Newsome 1998; Haider et al. 2006; Renart et al. 2007). In the balanced condition, fluctuations can drive the neuron to fire, thereby producing an irregular firing patterns. Equations governing the second-order correlations and covariances have been proposed and used to study balanced networks (Sejnowski 1976b; van Vreeswijk and Sompolinsky 1996, 1998; Moreno-Bote and Parga 2006; Soula and Chow 2007; El Boustani and Destexhe 2009). Similar approaches were also proposed for networks of binary neuron models (Hinton and Sejnowski 1983; Ackley et al. 1985; Ohira and Cowan 1993; Ginzburg and Sompolinsky 1994). A field-theoretic approach that generalizes the meanfield equations is also under way (Buice et al. 2009).

Other extensions include more complex intrinsic properties of neurons such as spike-frequency adaptation and bursting. In the simplest case, it is possible to obtain a second-order mean-field model by using a discretized time frame (Soula and Chow 2007), or in continuous-time (El Boustani and Destexhe 2009). The core of this approach is the "transfer function", which relates the output of the neuron (firing rate and its variance) to its inputs (mean rate and variance of 
excitatory and inhibitory inputs). Transfer functions can be estimated analytically using the Fokker-Planck approach (Tuckwell 1988; Abbott and van Vreeswijk 1993; Brunel and Sergi 1998; Brunel 2000; Fourcaud and Brunel 2002). However, such results apply only to continuous-time descriptions, and exact solutions are possible only for current-based models, which are not realistic and cannot take into account properties of neurons obtained from conductance measurements in vivo. More realistic transfer functions can be obtained from approximations (Kuhn et al. 2004) or numerical estimates (Kumar et al. 2008; Soula and Chow 2007). It should also be possible to estimate the transfer function directly from real cortical neurons in vitro using a dynamicclamp with injection of different combinations of conductance-based excitatory and inhibitory synaptic inputs (Bal and Destexhe, work in progress).

The derivation of the Wilson-Cowan model depended on taking the "thermodynamic limit" (in which the number of neurons approaches infinity), but finite-size effects in systems with a finite number of neurons can be important. This results in intermediate "mesoscopic" models that may be appropriate for modelling intermediate spatial scales, such as the number of neurons covered by a camera pixel in optical imaging, which ranges from hundreds to thousand of neurons (Brunel 2000; Renart et al. 2007; Soula and Chow 2007; El Boustani and Destexhe 2009). The extension of this approach to the spatial domain is a challenge for future studies.

To conclude, we have listed here just a few of the many theoretical developments that the Wilson-Cowan model has inspired. As experimental advances in recording from large populations of neurons are refined it will be possible to test the predictions of these models. Multi-scale models are now being developed that will integrate macroscopic models at large spatial scales with models at the microscopic scale, such as the Hodgkin-Huxley biophysical models of single neurons.

Open Access This article is distributed under the terms of the Creative Commons Attribution Noncommercial License which permits any noncommercial use, distribution, and reproduction in any medium, provided the original author(s) and source are credited.

\section{References}

Abbott LF, van Vreeswijk C (1993) Asynchronous states in networks of pulse-coupled oscillators. Phys Rev E 48:1483-1490

Ackley DH, Hinton GE, Sejnowski TJ (1985) A learning algorithm for Boltzmann machines. Cogn Sci 9:147-169

Amit DJ, Brunel N (1997) Model of global spontaneous activity and local structured activity during delay periods in the cerebral cortex. Cereb Cortex 7:237-252

Buice M, Cowan JD, Chow CC (2009) Generalized activity equations for neural networks. Neural Comput (in press)
Brunel N (2000) Dynamics of sparsely connected networks of excitatory and inhibitory spiking neurons. J Comput Neurosci 8:183208

Brunel N, Sergi S (1998) Firing frequency of leaky integrate-and-fire neurons with synaptic currents dynamics. J Theor Biol 195:87-95

El Boustani S, Destexhe A (2009) A master equation formalism for macroscopic modelling of asynchronous irregular activity states. Neural Comput 21:46-100

Fourcaud N, Brunel N (2002) Dynamics of the firing probability of noisy integrate-and-fire neurons. Neural Comput 14:2057-2110

Ginzburg I, Sompolinsky H (1994) Theory of correlations in stochastic neural networks. Phys Rev 50:3171-3191

Haider B, Duque A, Hasenstaub AR, McCormick DA (2006) Neocortical network activity in vivo is generated through a dynamic balance of excitation and inhibition. J Neurosci 26:4535-4545

van Hemmen JL (2004) Continuum limit of discrete neuronal structures: Is cortical tissue an "excitable" medium?. Biol Cybern 91:347-358

Hertz J, Lerchner A, Ahmadi M (2004) Mean field methods for cortical network dynamics. In: Érdi P et al (eds) Computational neuroscience: cortical dynamics. Springer, Berlin, pp 71-89

Hinton GE, Sejnowski TJ (1983) Optimal perceptual inference. In: Proceedings of the IEEE conference on computer vision and pattern recognition (CVPR). IEEE Computer Society, Washington DC, pp 448-453

Hopfield JJ (1982) Neural networks and physical systems with emergent collective computational abilities. Proc Natl Acad Sci 79:2554-2558

Hopfield JJ, Tank DW (1986) Computing with neural circuits: a model. Science 233:625-633

Kuhn A, Aertsen A, Rotter S (2004) Neuronal integration of synaptic input in the fluctuation-driven regime. J Neurosci 24:2345-2356

Kumar A, Schrader S, Aertsen A, Rotter S (2008) The high-conductance state of cortical networks. Neural Comput 20:1-43

Latham PE, Richmond BJ, Nelson PG, Nirenberg S (2000) Intrinsic dynamics in neuronal networks. I. Theory. J Neurophysiol 83:808827

Moreno-Bote R, Parga N (2006) Auto- and crosscorrelograms for the spike response of leaky integrate-and-fire neurons with slow synapses. Phys Rev Lett 96:028101

Ohira T, Cowan JD (1993) Master-equation approach to stochastic neurodynamics. Phys Rev E 48:2259-2266

Renart A, Moreno-Bote R, Wang XJ, Parga N (2007) Mean-driven and fluctuation-driven persistent activity in recurrent networks. Neural Comput 19:1-46

Sejnowski TJ (1976a) On global properties of neuronal interaction. Biol Cybern 22:85-95

Sejnowski TJ (1976b) On the stochastic dynamics of neuronal interaction. Biol Cybern 22:203-211

Shadlen MN, Newsome WT (1998) The variable discharge of cortical neurons: implications for connectivity, computation, and information coding. J Neurosci 18:3870-3896

Soula H, Chow CC (2007) Stochastic dynamics of a finite-size spiking neural network. Neural Comput 19:3262-3292

Tuckwell HC (1988) Introduction to theoretical neurobiology. Cambridge University Press, Cambridge

van Vreeswijk C, Sompolinsky H (1996) Chaos in neuronal networks with balanced excitatory and inhibitory activity. Science 274:1724-1726

van Vreeswijk C, Sompolinsky H (1998) Chaotic balanced state in a model of cortical circuits. Neural Comput 10:1321-1371

Wilson HR, Cowan JD (1972) Excitatory and inhibitory interactions in localized populations of model neurons. Biophys J 12:1-24

Wilson HR, Cowan JD (1973) A mathematical theory of the functional dynamics of nervous tissue. Kybernetik 13:55-80 